\title{
Distinct Visual Pathways Mediate Drosophila Larval Light Avoidance and Circadian Clock Entrainment
}

\author{
Alex C. Keene, ${ }^{1}$ Esteban 0. Mazzoni, ${ }^{1}$ Jamie Zhen, ${ }^{1}$ Meg A. Younger, ${ }^{1}$ Satoko Yamaguchi, ${ }^{1}$ Justin Blau, ${ }^{1}$ Claude Desplan, ${ }^{1}$ \\ and Simon G. Sprecher ${ }^{1,2}$ \\ ${ }^{1}$ Department of Biology, Center for Developmental Genetics, New York University, New York, New York 10003-6688, and 2Department of Biology, Institute \\ of Cell and Developmental Biology, University of Fribourg, 1700 Fribourg, Switzerland
}

Visual organs perceive environmental stimuli required for rapid initiation of behaviors and can also entrain the circadian clock. The larval eye of Drosophila is capable of both functions. Each eye contains only 12 photoreceptors (PRs), which can be subdivided into two subtypes. Four PRs express blue-sensitive rhodopsin5 (rh5) and eight express green-sensitive rhodopsin6 (rh6). We found that either PR-subtype is sufficient to entrain the molecular clock by light, while only the Rh5-PR subtype is essential for light avoidance. Acetylcholine released from PRs confers both functions. Both subtypes of larval PRs innervate the main circadian pacemaker neurons of the larva, the neuropeptide PDF (pigment-dispersing factor)-expressing lateral neurons (LNs), providing sensory input to control circadian rhythms. However, we show that PDF-expressing LNs are dispensable for light avoidance, and a distinct set of three clock neurons is required. Thus we have identified distinct sensory and central circuitry regulating light avoidance behavior and clock entrainment. Our findings provide insights into the coding of sensory information for distinct behavioral functions and the underlying molecular and neuronal circuitry.

\section{Introduction}

Animals detect and process a complex array of visual information from the environment. In both insects and vertebrates, visual information is perceived by photoreceptor (PR) neurons in the eye that express G-protein-coupled receptors, Rhodopsins, which transform light with specific wavelengths into neuronal information. This information is transmitted to second-order neurons for visual information processing before being transferred to higher brain centers. In both insects and vertebrates, the visual system processes information for rapid behaviors and entrains circadian pacemaker neurons.

The simple visual system of the Drosophila larva provides an excellent model to investigate these distinct roles. The larval eye (Bolwig organ) consists of four PRs expressing blue-sensitive rhodopsin5 (rh5) and eight PRs expressing green-sensitive rhodop-

Received Nov. 25, 2010; revised Feb. 25, 2011; accepted March 13, 2011.

Author contributions: A.C.K., E.O.M., J.B., C.D., and S.G.S. designed research; A.C.K., J.Z., M.A.Y., and S.G.S. performed research; A.C.K., S.Y., J.B., and S.G.S. contributed unpublished reagents/analytic tools; A.C.K. and S.G.S. analyzed data; A.C.K., J.B., C.D., and S.G.S. wrote the paper.

This work was funded by Grant EY013012 from the National Eye Institute-National Institutes of Health (NIH) to C.D., by Grant GM063911 from NIH to J.B., by the Swiss National Science Foundation, the Novartis Foundation, and the Janggen-Pöhn Stiftung (to S.G.S.), and by National Institute of General Medical Sciences National Research Service Award 5F32GM086207 to A.C.K., and was conducted in a facility constructed with the support of a Research Facilities Improvement Grant C06 RR-15518-01 from the National Center for Research Resources, NIH. We thankA. H. Brand, S. Britt, T. Cook, the Developmental Studies Hybridoma Bank, V. Hartenstein, F. Hirth, the Kyoto Stock Center, K. Matthews, M. Rosbash, the Vienna Drosophila RNAi Center for flies and antibodies, and Peter McKenney and June $\mathrm{Ng}$ for preliminary studies. We thank Scott Waddell (University of Massachusetts Medical School) for generously

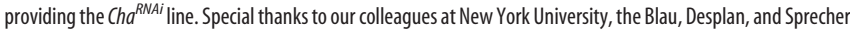
laboratories for fruitful discussions, to Mike Nitabach for advice on construction of the tethered $\alpha$-Btx transgene, and to Frank Pichaud (Medical Research Council, London).

Correspondence should be addressed to Simon G. Sprecher at the above address. E-mail: simon. sprecher@unifr.ch.

DOI:10.1523/JNEUROSCI.6165-10.2011

Copyright $\odot 2011$ the authors $\quad 0270-6474 / 11 / 316527-08 \$ 15.00 / 0$ sin6 (rh6) (Sprecher et al., 2007; Sprecher and Desplan, 2008). Thus, compared with the relative complexity of the visual system in the adult fly, only 12 neurons composed of two distinct cell types contribute to the perception of light. Axons from these 12 PRs innervate the larval optic neuropil (LON). The larval eye, along with the blue-sensitive photoreceptor cryptochrome (cry), regulates both entrainment of circadian rhythms and light avoidance (Mazzoni et al., 2005). Here we identify the neurotransmitter and PR-subtypes that mediate circadian entrainment and light avoidance. We further identify central brain neurons mediating rapid light avoidance.

\section{Materials and Methods}

Drosophila melanogaster strains and genetics. Flies and larvae were kept in $12 \mathrm{~h}$ light-dark (LD) cycles at $25^{\circ} \mathrm{C}$. For wild-type immunostainings we used $y w^{122}$ or $y w^{122}$;Sp/CyO;TM2/TM6b. In behavioral control experiments we used the genotype depicted in the corresponding figure and figure legend. We used the following mutants, Gal4 drivers, upstream activator sequence (UAS)-responder, and lacZ lines: $r h 5^{2}, r h 6^{1}$ (Yamaguchi et al., 2008); cry $^{b}$ (Mazzoni et al., 2005); rh5-Gal4, rh6-Gal4, GMRGal4, tim-Gal4, pdf-Gal4, Cha-Gal4, cry-Gal4, cry-Gal80, pdf-Gal80, UAS-hid, UAS-rpr, UAS-CD8::GFP (Bloomington Stock Center); UAS$S h i{ }^{T s l}$, UAS-H2B::YFP [anti-GFP (green fluorescent protein) antibody/ Biogenesis recognizes the yellow fluorescent protein (YFP) antigen] (Bellaïche et al., 2001); Cha-Gal80, rh5-lacZ, rh6-lacZ (Cook et al., 2003); hs-flp, UAS-FRT-CD2-STOP-FRT-CD8::GFP. UAS-Kir2.1 is UASmKir2.1(III), which has previously been described (Baines et al., 2001). For all experiments male and female larvae were used.

The UAS-Cha $a^{R N A i}$ construct consists of a P-element backbone harboring an inverted repeat of the Cha/VAChT locus cloned downstream of the Gal4 UAS-promoter. The inverted repeat is a 568 bp fragment targeting the first exon that is common to the Cha (choline acetyltransferase) and VAChT (vesicular ACh transporter) transcripts (Kitamoto et al., 1998). Cha is necessary for acetylcholine (ACh) synthesis, while VAChT 
is essential for transporting ACh into synaptic vesicles. Use of this AChRNAi (RNA interference) construct should block production of both of these proteins in specific cholinergic neurons and thereby impair ACh function.

The tethered UAS- $\alpha$-bungarotoxin (UAS- $\alpha$ Btx) 499 bp construct, was synthesized by GenScript with EcoRI and XhoI ends for insertion into pUAST (Brand and Perrimon, 1993). This construct contained the following: (1) Drosophila Kozak consensus sequence (CAAA), (2) secretion signal sequence and $\alpha$-bungarotoxin coding sequence (Ibañez-Tallon et al., 2004), (3) three repeats of a glycine-asparaginine linker (GN)3, (4) two copies of the myc-epitope tag, (5) (GN)9, and (6) Lynx1 C terminus for addition of glycosylphosphatidylinositol anchor for tethering to the membrane (Ibañez-Tallon et al., 2004). All of the protein-coding regions were codon-optimized for Drosophila (GenScript software).

Analysis of light-dependent clock entrainment. Light-entrained third instar larvae were exposed to a $2 \mathrm{~h}$ light pulse of 750 lux at Zeitgeber time 13 (ZT13), $1 \mathrm{~h}$ following the onset of lights-off. Light-entrained larvae without a light pulse were tested at ZT15 as controls. Larval brains were fixed for $15 \mathrm{~min}$ with $4 \%$ paraformaldehyde/PBS and subsequently stained as previously described (Blau and Young, 1999). TIM (circadian transcription factor Timeless) levels of stained brains were analyzed using confocal microscopy. One section at the largest diameter of 8-12 neurons was scanned for each genotype and condition. All experiments were repeated at least four times in independent experiments. Confocal settings were identical for all scans in each experiment. Staining intensity was defined by the mean pixel intensity within the cytoplasm minus the mean pixel intensity in the surrounding tissue (measured with the Leica confocal analysis software). The average ratio of pulsed/unpulsed was calculated for each genotype using Leica SP2 confocal processing software.

Immunohistochemistry and antibodies. Dissection and analysis of the brain and larval head skeleton were performed as previously described (Sprecher et al., 2007). Flip-out experiments were performed as described by Wong et al. (2002). Heat-shock was given in L1 larvae and dissections were performed in L3 larvae. Brains were mounted in Vectashield H-1000 (Vector Labs). Primary antibodies used were as follows: rabbit anti-Rh6, 1:10,000 (Tahayato et al., 2003); mouse anti-Rh5, 1:20, anti-Rh3, 1:20, or anti-Rh4 1:20 (Chou et al., 1996); mouse anti-Rh1, 1:20 [Developmental Studies Hybridoma Bank (DSHB)], mouse anti-Fasciclin II, 1:10 (Lin and Goodman, 1994); rat antiElav, 1:30 (DSHB); sheep anti-GFP, 1:1000 (Biogenesis); rabbit anti-PDF (pigment-dispersing factor), 1:50 (DSHB); mouse anti-Chp, 1:10 (DSHB); mouse anti- $\beta$ GAL, 1:20 (DSHB); and rat anti-TIM, 1:1000 (kindly provided by M. Rosbash, Brandeis University, Waltham, MA). Secondary goat antibodies were used for confocal microscopy conjugated with Alexa-488, Alexa-555, and Alexa-647 (Invitrogen), all at 1:300-1:500 dilution.

Laser confocal microscopy and image processing. Leica TCS SP2 and SP5 microscopes were used for all imaging. Spacing between optical sections ranged from 0.2 to $1.5 \mu \mathrm{m}$ and were recorded in "line average mode" with a picture resolution of $512 \times 512$ or $1024 \times 1024$ pixels. Captured images from optical sections were processed using Leica confocal software. Complete series of optical sections were imported and processed using ImageJ as previously described (Sprecher et al., 2006).

Behavioral assay and statistical analysis. We used the behavioral paradigm previously described by Mazzoni et al. (2005). Briefly, third instar larvae were grown in a $12 \mathrm{~h}$ dark-light cycle and tested for light avoidance between ZT2 and ZT4 at 750 lux. GraphPad Prism 5.0 was used for all statistical analysis. Each experimental set of data was statistically analyzed using an ANOVA with a Tukey's multiple-comparison post hoc test. Normal probability plots have been performed on random datasets of independent experiments supporting the normality assumption.

Eclosion experiments. Larvae grown in LD cycles on standard foodmedium were placed in eclosion monitors (Trikinetics) at the late wandering third instar during the light phase. Eclosion monitors were transferred to constant darkness (DD) $12 \mathrm{~h}$ later and eclosion was monitored $\sim 4 \mathrm{~d}$ later. Data represent the combined eclosions from $48 \mathrm{~h}$ of recordings collected in 4 independent experiments.

\section{Results}

\section{Rh5-PRs are required for light avoidance}

The larval eye is essential for light avoidance, as larvae with all PRs ablated are blind (Sawin-McCormack et al., 1995; Mazzoni et al., 2005). We used a standardized light avoidance assay in which larvae choose between 750 lux light and darkness. In this assay, $>70 \%$ of wild-type larvae robustly avoid light (Mazzoni et al., 2005). We assayed larvae carrying single mutations in $r h 5$ or $r h 6$, as well as rh5; rh6 double mutants, to determine which PR subsets are required for light avoidance. PRs maintain their normal projections and innervations of the LON in the absence of functional Rhodopsins (data not shown), rh5;rh6 double mutants were also completely blind (Fig. 1A). Light avoidance was also completely lost in $r h 5$ single mutants $(p<0.001)$. However, photophobicity was unaffected in $r h 6$ mutant larvae (Fig. $1 \mathrm{~A}$ ). Behavioral defects were observed as soon as $1 \mathrm{~min}$ after the start of the experiment and remained throughout the course of the $10 \mathrm{~min}$ assay (data not shown). These results indicate that $r h 5$ is required for light avoidance, while rh6 is dispensable.

To verify this finding, we genetically ablated each PR-subtype and assayed larvae for light avoidance. We coexpressed the proapoptotic genes head involution defective (hid) and reaper (rpr) in a subtype-specific manner, using either rh5-Gal4 or rh6-Gal4. Anti-Rh5, anti-Rh6 antibody staining confirmed that this manipulation fully and selectively ablated the targeted PRs (data not shown). Light avoidance was abolished in larvae with rh5 neurons ablated ( $r h 5>$ hid, rpr) (Fig. $1 B$ ) (ANOVA, $p<0.001$ ). However, $r h 6>$ hid, $r p r$ larvae robustly avoided light (Fig. $1 B$ ). These experiments confirm that only the Rh5-PR subtype is required for light avoidance, while the Rh6-PR subtype is dispensable.

\section{Either PR-subtype is sufficient for entrainment of the circadian clock}

In mammals, photoreceptors mediate vision while a distinct population of melanopsin-expressing retinal ganglion cells function to entrain circadian pacemaker cells via the retinohypothalamic tract (Hannibal et al., 2000; Hattar et al., 2002). PRs of the Drosophila larval eye innervate the lateral neurons (LNs) and are required to entrain the molecular clock (Mazzoni et al., 2005). The larval LNs survive metamorphosis and become the master pacemaker neurons in adult flies [small ventral LNs (LNvs)]. Furthermore, larval LNs probably maintain the circadian phase through metamorphosis (Sehgal et al., 1992; Kaneko et al., 1997). We therefore sought to identify the PR-subtype(s) responsible for entraining the central clock located in LNs.

The circadian transcription factor TIM is rapidly degraded upon light exposure, and TIM levels are a measure of the molecular response to light of the circadian clock. To investigate the role of larval PR-subtypes in clock entrainment, we examined TIM degradation after a $2 \mathrm{~h}$ light pulse beginning at ZT13, when TIM is normally localized to the cytoplasm. LNs also express cryptochrome (cry), which encodes an intrinsic blue lightsensitive photopigment that induces TIM degradation upon light exposure in the early morning (Stanewsky et al., 1998; Ceriani et al., 1999; Griffin et al., 1999; Klarsfeld et al., 2004). However, degradation of TIM in $c r y^{b}$ mutants was not significantly different from that in control animals with our $2 \mathrm{~h}$ light pulse protocol at ZT13 (Fig. 1E). This result is consistent with the fact that rhythmic cry expression is at low levels in the early evening (ZT13) (Stanewsky et al., 1998). Furthermore, since Cry protein itself is degraded by light, even low-level Cry protein accumulation would be minimal since larvae were only in darkness from ZT12 to ZT13. 
A

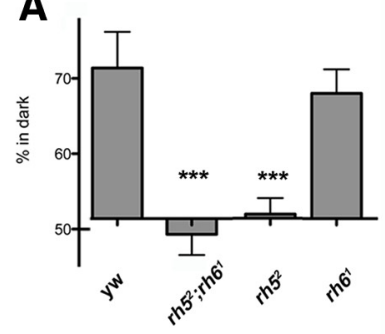

B

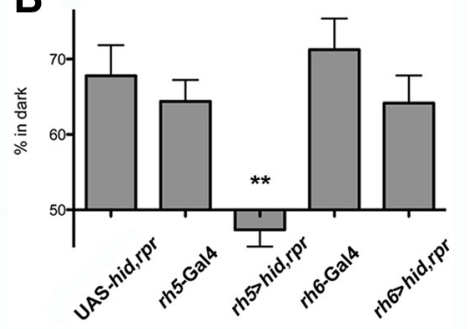

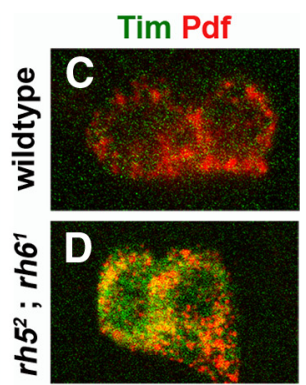
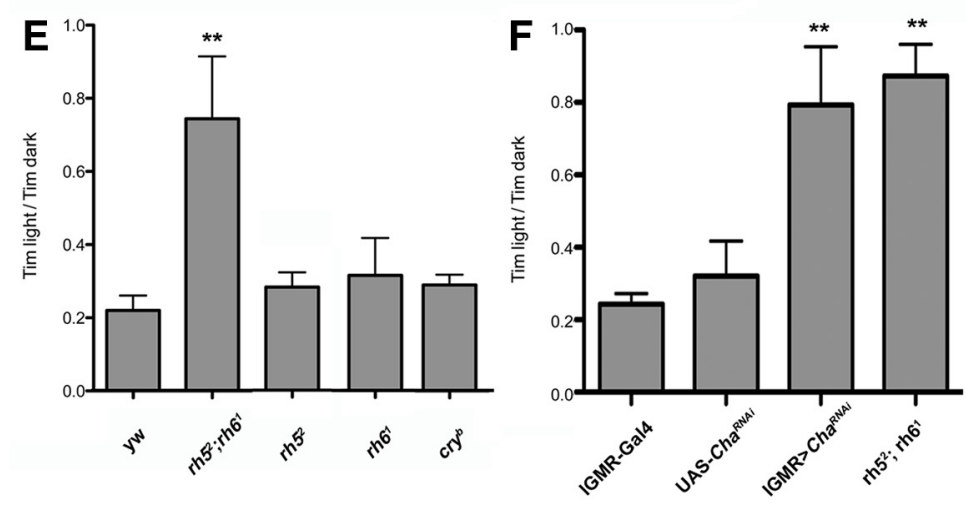

Figure 1. Function of Rh5-PRs and Rh6-PRs for light avoidance behavior and light-dependent TIM degradation in LNs. $\boldsymbol{A}$, $r h 5$;rh6 double-mutant, rh6 mutant, and $r h 5$ mutant larvae. yw (70.0\%; SEM, 4.7) and rh6 mutant $(66.6 \%$; SEM, 3.2) larvae were photophobic and did not differ from each other. rh5; rh6 double mutants (47.9\%; SEM, 2.7) and rh5 mutants (50.6\%; SEM, 2.1) both strongly reduced light avoidance compared with yw and rh6 mutant larvae ( $n=12$; ANOVA, $p<0.001)$. B, Genetic ablation of Rh5- or Rh6-PRs impairs light avoidance. In parental control strains rh5-Gal4 (67.8\%; SEM, 4.1), rh6-Gal4 (71.2\%; SEM, 4.1), and UAS-hid,rpr (67.8\%; SEM, 4.1), larval behavior was not significantly different, nor was the behavior of rh6-Gal4/UAS-hid,rpr (64.2\%; SEM, 3.7) larvae. In comparison, rh5-Gal4>UAS-hid,rpr (47.4\%; SEM 2.2) larvae were blind and significantly different from all other larvae $(n=10 ;$;NOVA, $p<0.001)$. C, $\boldsymbol{D}$, Confocal image of two LNs of a wild-type larva $(\boldsymbol{C})$ and a $r h 5$; rh6 double mutant $(\boldsymbol{D})$ after a $2 \mathrm{~h}$ light pulse stained with anti-PDF (red) and anti-TIM (green). TIM is degraded in wild-type larvae but remains present in rh5; rh6 double mutant. $\boldsymbol{E}$, Quantification of TIM levels in control, rh5; rh6 double-mutant, rh5 mutant, rh6 mutant, and $\mathrm{Cry}^{b}$ mutant larvae; $y$-axis represents the average ratio of [TIM pulsed]/[TIM nonpulsed]. rh5 mutants $(0.28$; SEM, 0.04), rh6 mutants $(0.31 ;$ SEM, 0.10$)$ and $c r y^{b}$ mutants $(0.29 ;$ SEM, 0.03$)$ are not significantly different from control animals, while $r h 5 ;$; $h 6$ double mutants $(0.74 ;$ SEM, 0.17$)$ were significantly different from all animals $(n=5$; ANOVA, $p<0.01)$. Error bars represent \pm SEM. $\boldsymbol{F}$, Quantification of TIM protein levels of larvae harboring either GMR-Gal4 or UAS-Ch ${ }^{R N A i}$ transgenes alone, rh5; rh6 double-mutant (control) larvae, and GMR $>$ Cha ${ }^{R N A i}$ larvae using confocal analysis. Parental GMR-Gal4 $(0.24 ;$ SEM, 0.03$)$ and UAS-Cha $a^{R N A i}(0.32 ;$ SEM, 0.09) lines are not significantly different from each other. TIM is not degraded in the $r h 5 ;$;rh6 double mutant $(0.79 ;$ SEM, 0.16$)$ and GMR $>C h a^{R N A i}(0.87 ;$ SEM, 0.06$)$ following light pulse, and the two lines are significantly different from GMR-Gal4 and UAS-Cha ${ }^{R N A i}$ lines $(n=4$; ANOVA, $p<0.01)$. Error bars represent \pm SEM.

Genetic ablation of all larval PRs blocks entrainment of the clock, presumably by inhibiting light-dependent degradation of TIM in LNs (Mazzoni et al., 2005). However, these findings are difficult to interpret because ablation of PRs also causes developmental abnormities in LNs (Malpel et al., 2002). To investigate the functional role of larval PR-subtypes in LN entrainment, we assayed light-induced TIM degradation in LNs of larvae mutant for $r h 5$, for $r h 6$, and for $r h 5 ; r h 6$ double mutants since LNs appear to develop normally in these mutants. In the absence of a light pulse, both wild-type and rh5;rh6 double-mutant larvae had high cytoplasmic TIM levels in LNs at ZT15. These findings suggest that the molecular clock still oscillates in blind animals, likely because it is entrained by Cry in the early morning. A $2 \mathrm{~h}$ light pulse significantly reduced TIM levels in LNs of wild-type larvae. However, TIM levels remained high in rh5; rh6 double mutants, indicating that the mutations render the molecular clock insensitive to light at this time of the day. Thus, the visual system is essential for TIM degradation by light in the early evening (Fig. $1 E$ ). We also analyzed TIM levels in $r h 5$ and $r h 6$ single mutants exposed to light. TIM levels responded to light like wild types and were reduced compared with rh5; rh6 double mutants after the light pulse (ANOVA; $p<0.01$ ). Therefore, while larval PRs are essential to confer TIM degradation in the early evening, either PR-subtype by itself is sufficient to entrain the clock.

We monitored eclosion in rh5;rh6 double mutants to determine whether the reduced TIM degradation in these mutants correlates with disrupted circadian entrainment. We placed control and mutant late wandering third instar larvae in constant darkness $2-3 \mathrm{~d}$ before eclosion to avoid interference by rhodopsins expressed in the developing adult eye just before eclosion. Interestingly, rh5;rh6 double mutants were similar to wild type, with the majority eclosing in the subjective day (data not shown). This is consistent with previous findings that ablation of the larval eye does not abolish circadian eclosion rhythms (Malpel et al., 2004). These findings suggest that cry alone is sufficient to support proper eclosion rhythms by entraining the clock in the early morning. Even though rh5;rh6 mutant larvae have decreased ability to degrade TIM in response to light, they are still capable of proper circadian behavior.

\section{Larval PR-subtypes project to distinct areas of the larval optic neuropil}

PRs of the larval eye extend their axons from the anterior part of the head skeleton into the LON (Fig. $2 A, C, D$ ), where Rh5and Rh6-PRs could achieve their unique function by signaling to different target neurons, and/or by signaling to the same neurons using distinct neurotransmitters. To investigate the axonal projection pattern of Rh5- and Rh6-PRs, we used rh5lac $Z$ and rh6-GFP reporters (Cook et al., 2003). We found that Rh5- and Rh6-PR projections occupy distinct regions of the LON: Rh5-PRs project medioventrally in the LON where they are surrounded by Rh6-PR termini (Fig. 2B) (data not shown). While the majority of synaptic connections localizes to the LON, we also observed Rh5 neurites bypassing the LON projecting to the adjacent ipsilateral central brain neuropil, indicating heterogeneous function of Rh5-PRs, as previously reported (Mazzoni et al., 2005) (data not shown).

\section{Both larval PR-subtypes are cholinergic}

Although PRs in the adult fly signal through the neurotransmitter histamine, larval PRs have been reported to be cholinergic (Gorczyca and Hall, 1987; Yasuyama et al., 1995). We investigated the neurotransmitter identity of larval PR-subtypes. Using an antihistamine antibody, we could not detect staining in the projections of larval PRs or any other area of the LON (data not shown). None of the neurotransmitter systems including serotonin, dopamine, octopamine, glutamate, and GABA were detected using neurotransmitter-specific antibodies or Gal4 lines (anti-serotonin, anti-dopamine, $d d c$-Gal4, Th1-Gal4, Tdc-Gal4, vGlut-Gal4, gad1Gal4) (data not shown). Instead, both larval PR-subtypes ex- 


\section{Elav Chp Fasll}
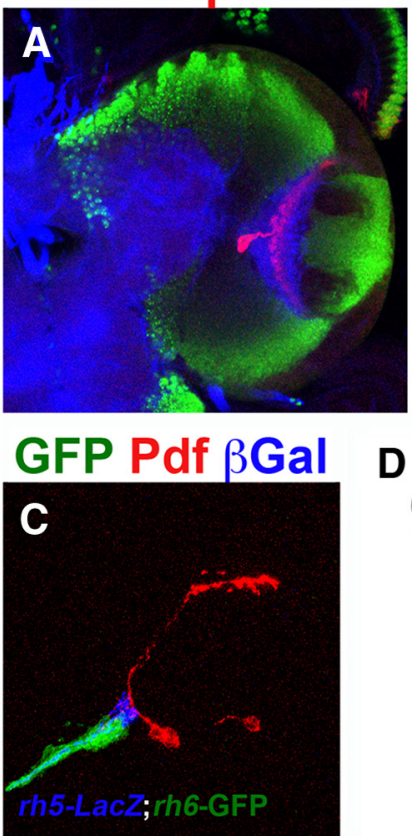

YFP Rh6 Rh5

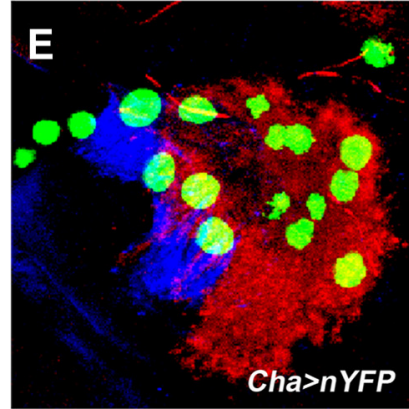

D
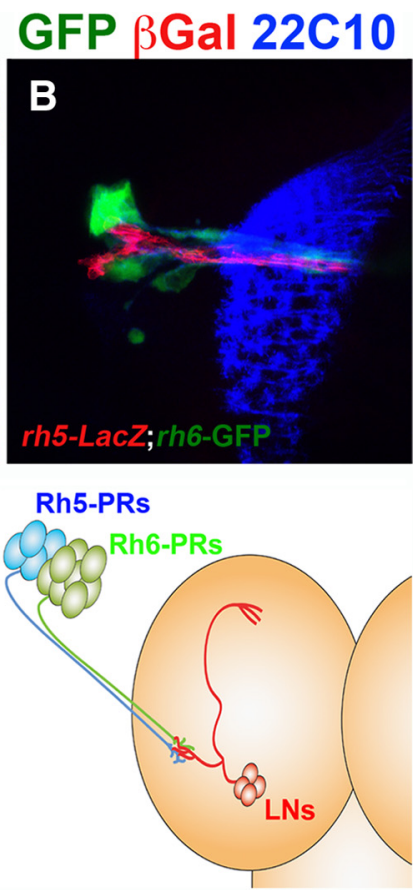

GFP Cha

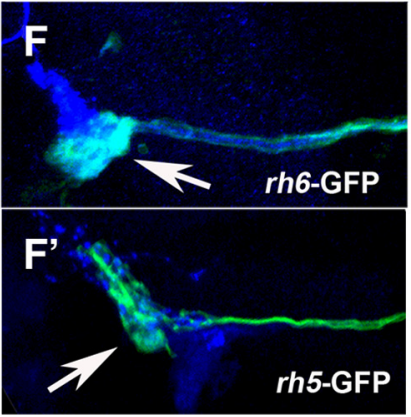

Figure 2. Rh5- and Rh6-PRs project to distinct domains and are cholinergic. $A$, Larval PR axons terminate in the LON located medial to the developing medulla neuropil. PR axons are labeled with anti-Chp (red), neuronal cell bodies of the brain and optic lobe neuropil are labeled with anti-Elav (green); anti-Fasciclin II (anti-FasII) (blue) labels PR axons and a subset of central brain axon fascicles. $\boldsymbol{B}$, Termini of PR-subtypes occupy distinct domains in the LON. Rh5 termini are labeled with $r h 5$ - LacZ (anti- $\beta$ Gal; red in $\boldsymbol{B}$, blue in $\boldsymbol{C}$ ) and Rh6 termini with $r h 6$-GFP (antiGFP; green in $\boldsymbol{B}, \boldsymbol{C}$. Both Rh5 and Rh6 PRs are marked with anti-22C10 in blue $(\boldsymbol{B})$. $\boldsymbol{C}, \mathrm{LN}$ dendrites arborize in the LON and overlap with both PR-subtypes. LNs are marked by anti-PDF (red). $\boldsymbol{D}$, Representation of the larval PR-subtypes and LNs within the LON. $\boldsymbol{E}$, Expression of UAS-H2B::YFP under the control of Cha-Gal4 labels the nuclei of Rh5- and Rh6-PRs (green, anti-YFP; red, anti-Rh6; blue, anti-Rh5). $\boldsymbol{F}, \boldsymbol{F}^{\prime}$, Double labeling of Rh6-axon termini (arrow) labeled with rh6-GFP, or Rh5-axon termini (arrow) labeled with rh5-GFP with anti-Cha (blue) and anti-GFP (green). Anti-Cha staining is detected in both PR-subtypes.

pressed choline acetyltransferase (Cha), the key enzyme in the biosynthesis of ACh, suggesting that $\mathrm{ACh}$ is the only classic neurotransmitter released from PRs. We used two independent experiments to assess whether both PR-subtypes were cholinergic. First, we expressed a UAS-Histone2B-YFP reporter under the control of Cha-Gal4 to label the nuclei of cholinergic neurons. We marked PRs with anti-Rh6 and anti-Rh5 antibodies and cholinergic neurons with anti-GFP (YFP is recognized by anti-GFP antibodies). All nuclei of Rh5- and Rh6-PRs are stained with anti-GFP, indicating that both PR-subtypes are cholinergic (Fig. $2 E$ ). Second, using rh5-lacZ or rh6-lacZ in combination with anti-Cha antibodies confirmed that Cha localizes to the axons of

projections to the LON in both PR-subtypes (Fig. $2 F, F^{\prime}$ ). Thus, both larval PR-subtypes are cholinergic.

\section{Acetylcholine is required in larval PRs for circadian clock resetting}

Larval LNs express a functional nicotinic ACh receptor, suggesting that they receive ACh input (Wegener et al., 2004). Therefore, $\mathrm{ACh}$ released from PRs may act to initiate TIM degradation and entrain the larval pacemaker neurons. We selectively disrupted ACh synthesis in PRs with Cha-RNAi to address whether ACh is required for light-dependent TIM degradation in LNs. TIM levels were measured after a light pulse as described above. Disrupting ACh synthesis in all PRs $\left(G M R>C h a^{R N A i}\right)$ blocked light-induced TIM degradation at ZT15. Although TIM levels in control larvae harboring the GMR-Gal4 or UAS-Cha ${ }^{R N A i}$ transgenes alone were not different from wild type, GMR $>C h a^{R N A i}$ larvae displayed high TIM protein levels (ANOVA, $p<0.01$ ) similar to $r h 5$; rh 6 double mutants (Fig. $1 F$ ). Therefore ACh functions in larval PRs for light-induced TIM degradation in pacemaker neurons.

\section{ACh release from Rh5-PRs drives light avoidance}

We sought to determine the role of ACh release from PRs in light avoidance. Inhibition of ACh synthesis in all PRs $\left(G M R>C h a^{R N A i}\right)$ abolished light avoidance (Fig. $3 A$ ) (ANOVA, $p<0.01$ ), suggesting that ACh released from larval PRs is required for light avoidance. We selectively disrupted ACh synthesis in Rh5- or Rh6-PRs to address the unique requirement of the $\mathrm{Rh} 5-\mathrm{PR}$ subtype in light avoidance. Inhibiting ACh function in Rh5-PRs $\left(r h 5>C h a^{R N A i}\right)$, but not in Rh6-PRs $\left(r h 6>C h a^{R N A i}\right)$, abrogated light avoidance (Fig. $3 A ; p<0.01$ ). Therefore Rh5-PRs mediate light avoidance through release of ACh.

To examine the role of PRs in light avoidance, we conditionally blocked synaptic transmission with UAS-Shibire ${ }^{T S I}\left(\mathrm{UAS}-\mathrm{Shi}^{T S I}{ }^{T \text { ). }}\right.$. Larvae in which UAS-Shi ${ }^{T S 1}$ is under the control of GMR-Gal4 avoided light normally at $20^{\circ} \mathrm{C}$, but they were blind at $30^{\circ} \mathrm{C}$ (ANOVA, $p<0.001$ ). We genetically rescued the GMR-Gal4induced blindness of UAS-Shi ${ }^{T S 1}$ using Cha-Gal80 to inhibit Gal4 function selectively in cholinergic cells (Kitamoto, 2002). Larvae in which GMR-Gal4 was inhibited with Cha-Gal80 had wild-type light avoidance at nonpermissive temperature (Fig. 3B).

Together with TIM-degradation experiments, our findings reveal that larval PRs confer both light entrainment of the circadian clock and rapid-light avoidance behavior. Both Rh5- and Rh6-PRs are capable of inducing TIM degradation in pacemaker neurons, while only Rh5-PRs are required for light avoidance. Both behaviors also require ACh produced by Rh5- and/or Rh6-PRs.

\section{Light avoidance requires acetylcholine reception in} clock neurons

To test the roles of LNs and other clock neurons in light avoidance, we assayed whether light avoidance requires reception of ACh by tim-expressing neurons (Mazzoni et al., 2005; Gong, 2009). We adopted a previously used strategy to inhibit ACh reception, and engineered a membrane-tethered $\alpha$-bungarotoxin $(\alpha-B t x)$ that was shown to block, in a cell-autonomous manner, the response of nicotinic acetylcholine receptors in Xenopus oocytes and zebrafish embryos (Ibañez-Tallon et al., 2004) (see Materials and Methods). We generated a line of flies containing UAS- $\alpha-B t x$, which was then crossed to a line containing the panneuronal elav-Gal4 driver to test its efficiency. Pan-neuronal expression of $\alpha-B t x$ resulted in larval lethality, presumably due to widespread blockade of cholinergic transmission (data not shown). Lethality has also been reported in a Chats mutant allele at non- 
A
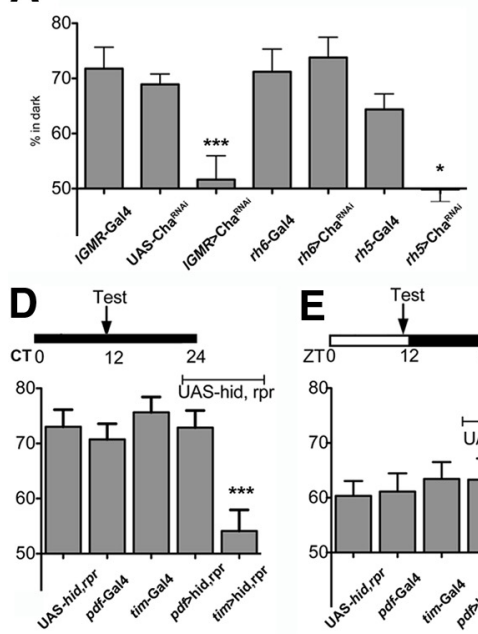
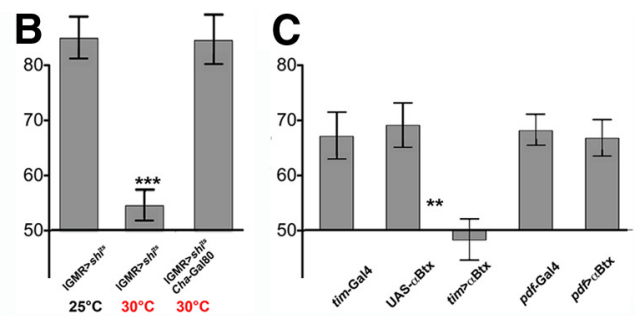

GFP Pdf NC82
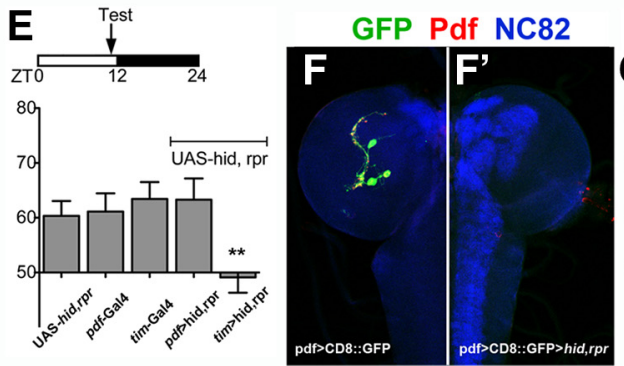

H

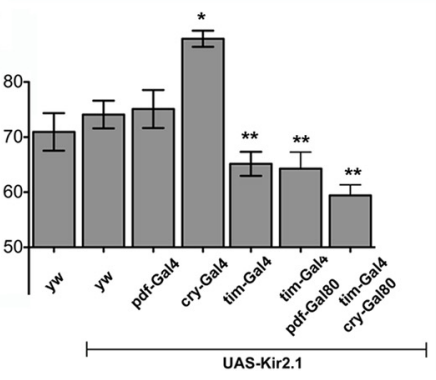

Figure 3. ACh from RH5-PRs and PDF-negative tim-expressing neurons are required for light avoidance behavior. $A$, Knockdown of ACh function in Rh5-PRs impairs light avoidance. The parental GMR-Gal4 (71.8\%; SEM, 3.9), UAS-Cha ${ }^{R N A i}(68.9 \%$; SEM, 1.9), rh6-Gal4 (71.3\%; SEM, 4.1), and rh5-Gal4 (64.4\%; SEM 2.8) larvae were not significantly different from each other or

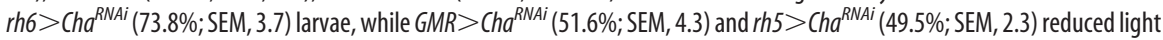
avoidance compared with single-transgene controls ( $n=10 ;$ ANOVA, $p<0.01)$. $\boldsymbol{B}$, Inclusion of the Cha-Gal 80 transgene rescues GMR-Gal4, UAS-Shi ${ }^{\text {TS1 }}$ larvae decreased light avoidance at $30^{\circ} \mathrm{C}(54.3 \% ; S E M, 2.7 ; n=11)$ compared with $25^{\circ} \mathrm{C}(71.8 \%$; SEM, 2.4;

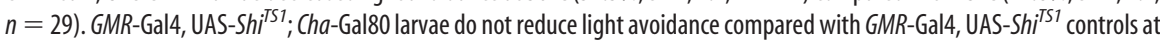
$25^{\circ} \mathrm{C}(83.9 \%$; SEM 4.3; $n=8)$. C, Disrupting ACh input in all clock neurons (LNs, DN1s and DN2s) impairs light avoidance. Control larvae harboring tim-Gal4 (65.2\%; SEM, 3.7; $n=8$ ) or UAS- $\alpha$-Btx transgenes (67.0\%; SEM 3.5; $n=10$ ) had greater light avoidance than $\operatorname{tim}>\alpha$-Btx larvae (48.4\%; SEM 3.3; $n=9)$. Light avoidance in $p d f-G a l 4(66.1 \% ;$ SEM $2.4 ; n=8)$ and $p d f>\alpha-B t x$ larvae (64.8\%; SEM 2.9; $n=9$ ) did not differ from that of other controls. (ANOVA; $p<0.01$ ). $\boldsymbol{D}, \boldsymbol{E}$, Larvae were tested in LD (D) or DD (E). LN-ablated larvae did not differ from wild type at either ZT24 or CT24, while tim-Gal4 > UAS-hid, UAS-rpr larvae reduced light avoidance compared with single-transgene controls and larvae with ablated LNs ( $p d f>$ hid, rpr) (ANOVA, $p<0.01 ; n=10)$. $\boldsymbol{F}, \boldsymbol{F}^{\prime}$, pdf-Gal4 labels LNs $(\boldsymbol{F})$ and expression of UAS-hid, rpr by pdf-Gal4 ( $\left.\boldsymbol{F}^{\prime}\right)$ ablates the LNs; labeled with $p d f>$ CD8::GFP (green) and anti-PDF (red) $\left(\boldsymbol{F} ; p d f>\right.$ CD8::GFP; $\boldsymbol{F}^{\prime}, p d f>$ CD8::GFP $>$ hid, $\left.r p r\right)$. G, Larvae with silenced LNs ( $p d f>$ Kir2.1) avoided light at wild-type levels (73.3\%; SEM, 2.8; $n=10$ ). $\boldsymbol{H}$, Larvae with silenced DN2 and fifth LN silenced (tim-Gal4,cry-Gal80;UAS-Kir2.1) display strongly reduced light avoidance $(60.4 \% ; S E M, 2.4 ; n=12)$ compared with Gal 4 or UAS-responder lines $(n=12)$. Error bars represent \pm SEM.

permissive temperature (Kitamoto et al., 1992). Larvae expressing $\alpha$-Btx in all clock neurons ( $t$ im-Gal4 $>\alpha$-Btx) were blind (Fig. $3 C$ ) (ANOVA, $p<0.001$ ). In contrast, larvae expressing UAS- $\alpha$ $B t x$ in only LNs ( $p d f>\alpha$-Btx) did not differ significantly from the parental $p d f$-Gal4 or UAS- $\alpha$-Btx animals (Fig. $3 C$ ). Thus, reception of input from cholinergic neurons is required for light avoidance in tim-expressing neurons, but not in the PDFexpressing LNs.

\section{PDF neurons are dispensable for light avoidance}

Larval neurons expressing circadian clock genes comprise the four PDF-expressing LNs, a fifth PDF-negative LN, and two pairs of dorsal neurons called DN1s and DN2s (Kaneko and Hall, 2000). All of these neurons express tim. We had previously reported that the circadian LNs act downstream of the larval eye to mediate light avoidance (Mazzoni et al., 2005). However, using a

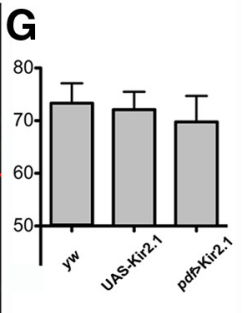

different assay, another group reported that larvae with ablated LNs display normal photophobic behavior (Hassan et al., 2005). In addition, we described above that $\mathrm{ACh}$ reception by $\mathrm{LNs}$ is not required for light avoidance, whereas ACh signaling from Rh5-PRs is required.

To conclusively determine whether LNs are required for light avoidance, we used UAS-hid and $r p r$ to ablate all circadian clock neurons ( $t$ im-Gal4), or only LNs ( $p d f$-Gal4; Fig. $3 F$ ). Expression of hid and rpr with timGal4 or $p d f$-Gal4 efficiently ablated either class of neurons (Fig. 3F'; data not shown). To functionally test the role of these neurons, larvae were entrained to LD cycles and assayed for light avoidance, either when larvae had been exposed to light for $12 \mathrm{~h}$ (at ZT12), or after being transferred to DD, and assayed at the end of the second day in $\mathrm{DD}$ [circadian time 12 (CT12)]. Consistent with our previous report, larvae in DD had higher light avoidance scores than larvae taken from LD (Mazzoni et al., 2005) ( $p<$ 0.001 ). This is presumably due to desensitization of the visual system by light (Mazzoni et al., 2005). However, in contrast to our previous report (Mazzoni et al., 2005), we found no effect of LN ablation on light avoidance: $\mathrm{LN}$-ablated larvae ( $p d f>$ hid, $r p r$ ) displayed wild-type photophobic behavior at both ZT12 and CT12 ( $p>0.85, p>$ 0.97 ) (Fig. $3 D, E$ ). Ablation of all clock neurons with tim $>$ hid, rpr severely disrupted light avoidance compared with larvae harboring UAS-hid, UAS-rpr, or tim-Gal4 transgenes alone, and PDF-expressing LN-ablated larvae ( $p d f>$ hid,rpr; $p<$ 0.001) (Fig. 3D,E). Light avoidance of tim > hid, rpr was not different in LD compared with DD conditions (Fig. 3D,E). Therefore tim-expressing neurons are required for light avoidance, while $p d f$ expressing LNs are dispensable.

Next, we addressed the subpopulation of tim-expressing neurons mediating light avoidance. We silenced distinct populations of tim neurons with the inward-rectifying $\mathrm{K}^{+}$channel mKir2.1 to circumvent potential developmental problems by genetic ablation experiments and to preserve the integrity of the neuronal circuit. As expected, silencing all tim neurons with mKir2.1 disrupted light avoidance, while larvae expressing UASmKir2.1 in PDF-expressing LNs avoided light as well as wild type (Fig. 3G), confirming that these neurons are not required for light avoidance. To definitively confirm that light avoidance defects in larvae with disrupted tim neurons were not due to LN function, we prevented expression of $m$ Kir2.1 in LNs using pdf-Gal80 (timGal4, $p d f$-Gal80, UAS-mKir2.1). These larvae had reduced light avoidance (Fig. $3 G$ ). No behavioral alterations were observed by the expression of Gal80 alone (data not shown). In larvae, cry is expressed in all tim-expressing neurons except the DN2 cluster and the fifth LN (Kitamoto, 2002). cry-Gal4, UAS-mKir2.1 larvae did not show reduced light avoidance but, instead, increased light 

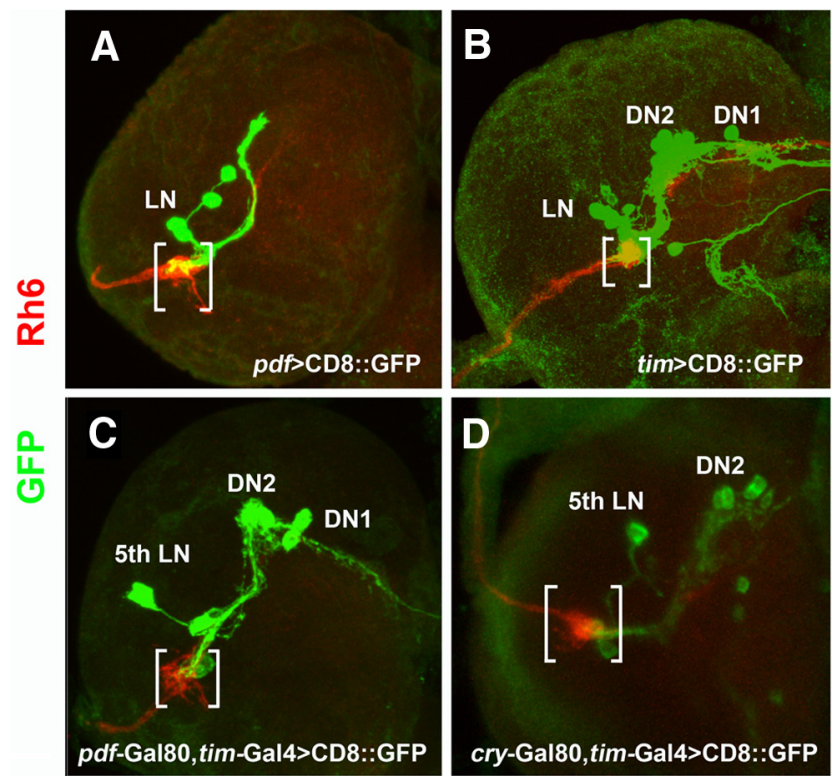

hs-flp; tim-Gal4/ UAS>CD2,STOP>CD8::GFP
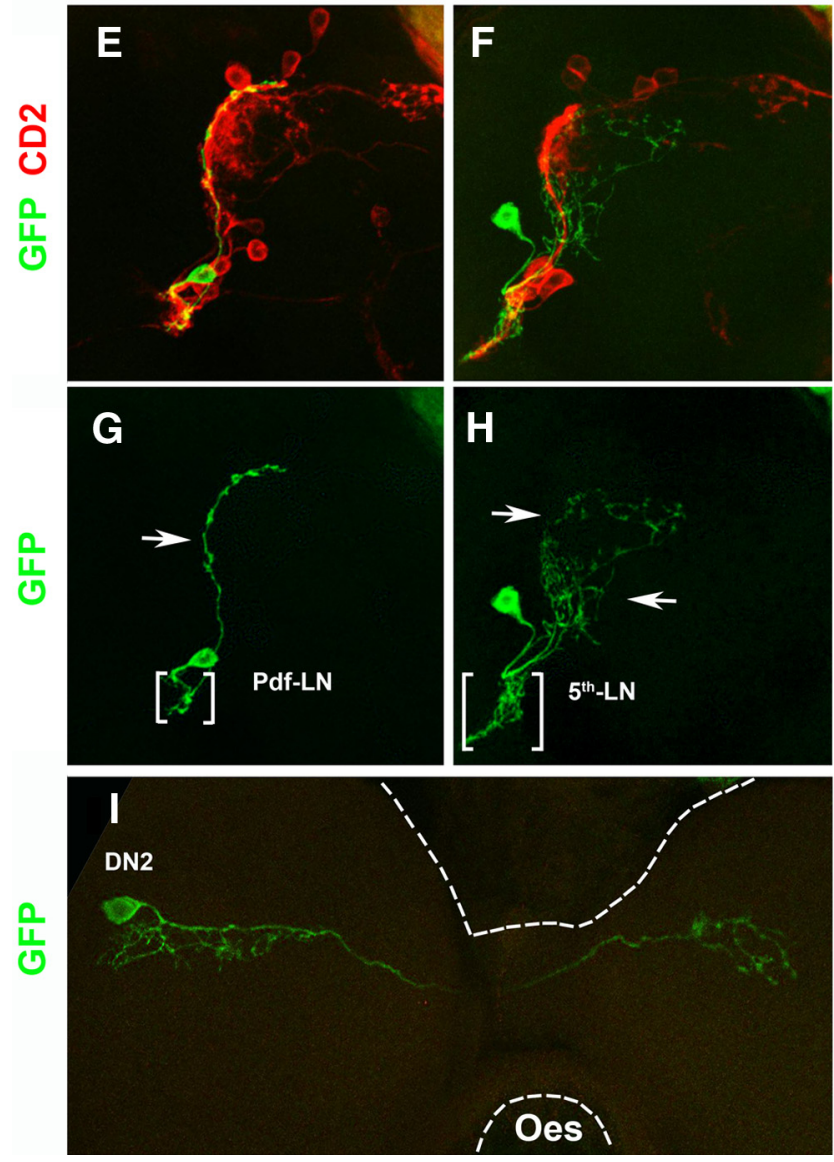

Figure 4. PDF-expressing LNs and fifth $L N$ innervate the LON, while DN2s do not. $\boldsymbol{A}-\boldsymbol{D}$, Projection pattern of the neurons expressing UAS-CD8::GFP under the control of $p d f-G a 14(\boldsymbol{A}$; labeling four LNs), tim-Gal4 ( $\boldsymbol{B}$; labeling all LNs, DN2s, and DN1s), tim-Gal4, pdf-Gal80 (C; labeling the fifth LN, DN2s, and DN1s), tim-Gal4, cry-Gal80 (D; labeling the fifth LN and DN2s). Anti-GFP in green, projections of Rh6-PRs showing the LON region labeled with anti-Rh6 in red. $E-I$, Genetic flip-out experiments showing anatomy of individual neurons generated with hsflp; tim-Gal4/UAS-FRT-CD2-STOP-FRT-CD8::GFP (anti-GFP in green, anti-CD2 in red). E, G, PDFexpressing LNs connect to the LON and display a typical axonal extension toward the dorsal protocerebrum (arrow). $\boldsymbol{F}, \boldsymbol{H}$, The fifth $L N$ innervates the $L O N$ and shows a very distinct projection pattern with various ramifications toward the central brain (arrows). $I$, The DN2 neuron avoidance, suggesting that DN1s normally reduce photophobicity (addressed further by Collins and Blau, unpublished observation). To address the possible role of DN2s and the fifth LN in light avoidance, we used the tim-Gal4,cry-Gal80 combination. Interestingly tim-Gal4,cry-Gal80, UAS-mKir2.1 larvae exhibited reduced light avoidance (Fig. $3 H$ ). Therefore, DN2 neurons and/or the fifth LN, but not PDF-expressing LNs and DN1 neurons, are required for normal light avoidance.

To gain deeper insight into the functional relationship between larval PRs and their target clock neurons, we used confocal microscopy to assess their gross anatomy. $p d f$-Gal4 drove expression in four LNs with dendritic arborizations in the LON. Comparably, tim-Gal4 drove expression in all clock neurons, including all 5 LNs whose projections innervate the LON (Fig. 4B). In timGal4, $p d f$-Gal80 larvae, only a single LN cell body was observed, but PR termini still made apparent contacts with GFP-labeled LN projections, suggesting that larval PRs connect to the non-PDFexpressing fifth LN (Fig. 4C; data not shown). This innervation was also detected when GFP was expressed with tim-Gal4,cryGal80 that only labels the fifth LN and DN2 neurons (Fig. 4D). Thus, in all cases, dendritic arborizations of clock neurons into the LON were observed.

To further distinguish innervation of PDF-expressing LNs, the fifth LN and DN2s, we assessed their anatomical properties using flip-out experiments (Wong et al., 2002). As previously described, we found that the PDF-expressing LNs innervate the LON and project into a distinct domain of the dorsal protocerebrum (HelfrichFörster, 1997; Helfrich-Förster et al., 2002; Malpel et al., 2002) (Fig. $4 E, G$ ). In agreement with previous anatomical analysis (HelfrichFörster et al., 2007), single-cell analysis of the fifth LN revealed prominent innervation of the LON with a broader projection domain than PDF-expressing LNs (Fig. 4F, H). Consistent with previously published data (Kaneko and Hall, 2000; Shafer et al., 2006), we observed that the DN2s project to the contralateral side of the brain, but do not show dendritic arborizations in the LON (Fig. 4I). Together, these findings suggest that PR projections not only contact PDF-expressing LNs, but also connect with other secondorder neurons within the LON (likely the fifth LNs) that might mediate light avoidance.

\section{Discussion}

Genetic silencing and ablation experiments revealed that larval Rh5-PRs in combination with the fifth LN and DN2s are essential for rapid photophobic behavior. rh 5 mutants and genetic ablation of Rh5-PRs strongly reduced light avoidance. In addition, selectively silencing the fifth LN and DN2s by expressing UASmKir2.1 in tim-Gal4;cry-Gal80 larvae disrupts light avoidance. Identifying direct Rh5-PR target neurons within the LON will further help our understanding of the circuitry underlying light avoidance. These second-order neurons may either include or signal to the fifth LN and/or the DN2 clock neurons that we have implicated in light avoidance. The connectivity of fifth LN with PRs makes it a prime candidate for directly mediating light avoidance.

Our results raise the possibility of distinct roles for PDFpositive and PDF-negative LNs, where PDF-positive LNs drive circadian behavior, while PDF-negative LNs (fifth LN) would be

does not show innervation of the LON; it projects its axon toward the contralateral brain hemisphere (dashed line shows the border of the brain hemispheres; Oes delimits the esophageal opening). Brackets in $\boldsymbol{A}-\boldsymbol{D}, \boldsymbol{G}$, and $\boldsymbol{H}$ show the LON region. 
critical for processing light cues for avoidance behavior. Adult flies have distinct types of LNvs, large (l-LNvs) and small (sLNvs), both of which express PDF and appear to have divergent functions. The PDF-positive LNvs appear to be the primary pacemaker cells in DD and regulate the morning activity peak in LD, while the fifth PDF-negative s-LNvs and some PDF-negative dorsal LNs, an adult-specific LN population, help regulate the evening activity peak in LD (Rieger et al., 2006).

To date, the connectivity of DN1 and DN2 neurons with the fifth LN and larval PRs remains unclear. It has recently been shown that explorative head-swinging behavior in response to a light pulse in late third instar larvae requires serotonergic neurons (Rodriguez Moncalvo and Campos, 2009). Dendritic arbors of serotonergic neurons in the LON dramatically increase during larval life. Interestingly, dendritic growth of these serotonergic neurons requires Rh6-PRs. Furthermore, serotonergic projections are in close proximity to the DNs and fifth LN (Hamasaka and Nässel, 2006). Therefore, it seems likely that Rh6-PRs function in combination with these serotonergic neurons for lightdependent behaviors (Rodriguez Moncalvo and Campos, 2005). It will be of great interest to investigate whether serotonin neurons functionally connect Rh5-PRs to the DN2 neurons or form connections in the LON with the fifth LN. It is possible that functional connections between serotonergic neurons and the clock neurons are required for light avoidance. Closely examining the neuroanatomy of LON-innervating neurons may reveal the neural connectivity by which larval PRs or the fifth LN communicates with DN1/DN2 neurons to regulate light avoidance. In addition, future work will examine the synaptic targets of Rh5PRs that bypass the LON.

Either Rh5-PRs or Rh6-PRs are sufficient for normal cycling of gene expression within the larval pacemaker neurons. Disrupting the function of both $r h 5$ and $r h 6$ results in larvae that are less sensitive to TIM degradation-induced light pulses, but are still able to show normal eclosion patterns. Normal eclosion in the absence of the larval eye is likely mediated by cry function in the LNs. Future experiments examining eclosion patterns in rh5; rh6; cry triple mutants will be informative in revealing functional redundancy within this system. Together, our results reveal how both redundant and distinct PR connectivity modulates two visual behaviors. Larval PRs confer both light-dependent entrainment of the circadian clock and rapid-light avoidance behavior. Interestingly, the function for rapid-light behaviors already differs at the level of the PR-subtypes in the eye. While the Rh5-subtype is essential for rapid-light avoidance, the Rh6subtype is dispensable.

Clock entrainment and rapid-light avoidance require ACh release from PRs. We have developed genetically expressed $\alpha$-bungarotoxin as a tool for probing ACh function in neural populations and find that expression of $\alpha$-Btx in all circadian neurons disrupts light avoidance. Future work refining the sites of ACh function in light avoidance should identify specific populations of central brain neurons regulating light avoidance. We have not been able to reproduce the results showing loss of photophobic behavior after ablation of PDF neurons reported in Mazzoni et al. (2005). Instead, we find that the circadian PDFexpressing LNs are dispensable for light avoidance, consistent with findings of Hassan et al. (2005). Further analysis of the timexpressing neurons regulating light avoidance revealed a possible role for DN2 neurons and/or that the fifth $\mathrm{LN}$ is required for this behavior. It is likely that Rh5-PR neurons have LON targets in addition to PDF-expressing LNs that signal light avoidance.
Therefore, we have also identified divergence in the central neurons mediating visual and circadian behaviors.

\section{Notes}

Supplemental material for this article is available at http://www.unifr.ch/ zoology/eng/home/research-groups/sprecher/sprecherpub. The supplemental material consists of five figures and the corresponding figure legends: (1) Projection pattern of PRs in rh5, rh6 double mutants, projections of Rh5-PRs to the deeper brain; (2) Time course analysis of photobehavior of $y w, r h 6, r h 5$, and rh5;rh6 double mutants; (3) Eclosion rhythms of wild-type, $r h 6, r h 5$, and $r h 5$; rh6 double mutants; (4) Connection of larval PRs to the 5th LN; and (5) Working model for the distinct roles of larval PR-subtypes. This material has not been peer reviewed.

\section{References}

Baines RA, Uhler JP, Thompson A, Sweeney ST, Bate M (2001) Altered electrical properties in Drosophila neurons developing without synaptic transmission. J Neurosci 21:1523-1531.

Bellaïche Y, Gho M, Kaltschmidt JA, Brand AH, Schweisguth F (2001) Frizzled regulates localization of cell-fate determinants and mitotic spindle rotation during asymmetric cell division. Nat Cell Biol 3:50-57.

Blau J, Young MW (1999) Cycling vrille expression is required for a functional Drosophila clock. Cell 99:661-671.

Brand AH, Perrimon N (1993) Targeted gene expression as a means of altering cell fates and generating dominant phenotypes. Development 118:401-415.

Ceriani MF, Darlington TK, Staknis D, Más P, Petti AA, Weitz CJ, Kay SA (1999) Light-dependent sequestration of TIMELESS by CRYPTOCHROME. Science 285:553-556.

Chou WH, Hall KJ, Wilson DB, Wideman CL, Townson SM, Chadwell LV, Britt SG (1996) Identification of a novel Drosophila opsin reveals specific patterning of the R7 and R8 photoreceptor cells. Neuron 17: 1101-1115.

Cook T, Pichaud F, Sonneville R, Papatsenko D, Desplan C (2003) Distinction between color photoreceptor cell fates is controlled by Prospero in Drosophila. Dev Cell 4:853-864.

Gong Z (2009) Behavioral dissection of Drosophila larval phototaxis. Biochem Biophys Res Commun 382:395-399.

Gorczyca MG, Hall JC (1987) Immunohistochemical localization of choline acetyltransferase during development and in Chats mutants of Drosophila melanogaster. J Neurosci 7:1361-1369.

Griffin EA Jr, Staknis D, Weitz CJ (1999) Light-independent role of CRY1 and CRY2 in the mammalian circadian clock. Science 286:768-771.

Hamasaka Y, Nässel DR (2006) Mapping of serotonin, dopamine, and histamine in relation to different clock neurons in the brain of Drosophila. J Comp Neurol 494:314-330.

Hannibal J, Møller M, Ottersen OP, Fahrenkrug J (2000) PACAP and glutamate are co-stored in the retinohypothalamic tract. J Comp Neurol 418:147-155.

Hassan J, Iyengar B, Scantlebury N, Rodriguez Moncalvo V, Campos AR (2005) Photic input pathways that mediate the Drosophila larval response to light and circadian rhythmicity are developmentally related but functionally distinct. J Comp Neurol 481:266-275.

Hattar S, Liao HW, Takao M, Berson DM, Yau KW (2002) Melanopsincontaining retinal ganglion cells: architecture, projections, and intrinsic photosensitivity. Science 295:1065-1070.

Helfrich-Förster C (1997) Development of pigment-dispersing hormoneimmunoreactive neurons in the nervous system of Drosophila melanogaster. J Comp Neurol 380:335-354.

Helfrich-Förster C, Edwards T, Yasuyama K, Wisotzki B, Schneuwly S, Stanewsky R, Meinertzhagen IA, Hofbauer A (2002) The extraretinal eyelet of Drosophila: development, ultrastructure, and putative circadian function. J Neurosci 22:9255-9266.

Helfrich-Förster C, Shafer OT, Wülbeck C, Grieshaber E, Rieger D, Taghert P (2007) Development and morphology of the clock-gene-expressing lateral neurons of Drosophila melanogaster. J Comp Neurol 500:47-70.

Ibañez-Tallon I, Wen H, Miwa JM, Xing J, Tekinay AB, Ono F, Brehm P, Heintz N (2004) Tethering naturally occurring peptide toxins for cellautonomous modulation of ion channels and receptors in vivo. Neuron 43:305-311.

Kaneko M, Hall JC (2000) Neuroanatomy of cells expressing clock genes in Drosophila: transgenic manipulation of the period and timeless genes to 
mark the perikarya of circadian pacemaker neurons and their projections. J Comp Neurol 422:66-94.

Kaneko M, Helfrich-Förster C, Hall JC (1997) Spatial and temporal expression of the period and timeless genes in the developing nervous system of Drosophila: newly identified pacemaker candidates and novel features of clock gene product cycling. J Neurosci 17:6745-6760.

Kitamoto T (2002) Conditional disruption of synaptic transmission induces male-male courtship behavior in Drosophila. Proc Natl Acad Sci U S A 99:13232-13237.

Kitamoto T, Ikeda K, Salvaterra PM (1992) Analysis of cis-regulatory elements in the $5^{\prime}$ flanking region of the Drosophila melanogaster choline acetyltransferase gene. J Neurosci 12:1628-1639.

Kitamoto T, Wang W, Salvaterra PM (1998) Structure and organization of the Drosophila cholinergic locus. J Biol Chem 273:2706-2713.

Klarsfeld A, Malpel S, Michard-Vanhée C, Picot M, Chélot E, Rouyer F (2004) Novel features of cryptochrome-mediated photoreception in the brain circadian clock of Drosophila. J Neurosci 24:1468-1477.

Lin DM, Goodman CS (1994) Ectopic and increased expression of Fasciclin II alters motoneuron growth cone guidance. Neuron 13:507-523.

Malpel S, Klarsfeld A, Rouyer F (2002) Larval optic nerve and adult extraretinal photoreceptors sequentially associate with clock neurons during Drosophila brain development. Development 129:1443-1453.

Malpel S, Klarsfeld A, Rouyer F (2004) Circadian synchronization and rhythmicity in larval photoperception-defective mutants of Drosophila. J Biol Rhythms 19:10-21.

Mazzoni EO, Desplan C, Blau J (2005) Circadian pacemaker neurons transmit and modulate visual information to control a rapid behavioral response. Neuron 45:293-300.

Rieger D, Shafer OT, Tomioka K, Helfrich-Förster C (2006) Functional analysis of circadian pacemaker neurons in Drosophila melanogaster. J Neurosci 26:2531-2543.

Rodriguez Moncalvo VG, Campos AR (2005) Genetic dissection of trophic interactions in the larval optic neuropil of Drosophila melanogaster. Dev Biol 286:549-558.

Rodriguez Moncalvo VG, Campos AR (2009) Role of serotonergic neurons in the Drosophila larval response to light. BMC Neurosci 10:66.
Sawin-McCormack EP, Sokolowski MB, Campos AR (1995) Characterization and genetic analysis of Drosophila melanogaster photobehavior during larval development. J Neurogenet 10:119-135.

Sehgal A, Price J, Young MW (1992) Ontogeny of a biological clock in Drosophila melanogaster. Proc Natl Acad Sci U S A 89:1423-1427.

Shafer OT, Helfrich-Förster C, Renn SC, Taghert PH (2006) Reevaluation of Drosophila melanogaster's neuronal circadian pacemakers reveals new neuronal classes. J Comp Neurol 498:180-193.

Sprecher SG, Desplan C (2008) Switch of rhodopsin expression in terminally differentiated Drosophila sensory neurons. Nature 454:533-537.

Sprecher SG, Urbach R, Technau GM, Rijli FM, Reichert H, Hirth F (2006) The columnar gene vnd is required for tritocerebral neuromere formation during embryonic brain development of Drosophila. Development 133: 4331-4339.

Sprecher SG, Pichaud F, Desplan C (2007) Adult and larval photoreceptors use different mechanisms to specify the same Rhodopsin fates. Genes Dev 21:2182-2195.

Stanewsky R, Kaneko M, Emery P, Beretta B, Wager-Smith K, Kay SA, Rosbash M, Hall JC (1998) The cryb mutation identifies cryptochrome as a circadian photoreceptor in Drosophila. Cell 95:681-692.

Tahayato A, Sonneville R, Pichaud F, Wernet MF, Papatsenko D, Beaufils P, Cook T, Desplan C (2003) Otd/Crx, a dual regulator for the specification of ommatidia subtypes in the Drosophila retina. Dev Cell $5: 391-402$.

Wegener C, Hamasaka Y, Nässel DR (2004) Acetylcholine increases intracellular Ca2 + via nicotinic receptors in cultured PDF-containing clock neurons of Drosophila. J Neurophysiol 91:912-923.

Wong AM, Wang JW, Axel R (2002) Spatial representation of the glomerular map in the Drosophila protocerebrum. Cell 109:229-241.

Yamaguchi S, Wolf R, Desplan C, Heisenberg M (2008) Motion vision is independent of color in Drosophila. Proc Natl Acad Sci U S A 105: $4910-4915$

Yasuyama K, Kitamoto T, Salvaterra PM (1995) Localization of choline acetyltransferase-expressing neurons in the larval visual system of Drosophila melanogaster. Cell Tissue Res 282:193-202. 